\title{
Earlier re-initiation of enteral feeding after necrotizing enterocolitis decreases recurrence or stricture: a systematic review and meta- analysis
}

\author{
Ekta U. Patel ${ }^{1} \cdot$ Dulaney A. Wilson ${ }^{2} \cdot$ Emily A. Brennan $\mathbb{1}^{3} \cdot$ Aaron P. Lesher $\mathbb{1}^{4} \cdot$ Rita M. Ryan ${ }^{5}$
}

Received: 6 January 2020 / Revised: 4 June 2020 / Accepted: 7 July 2020 / Published online: 18 July 2020

(c) The Author(s), under exclusive licence to Springer Nature America, Inc. 2020

\begin{abstract}
Objective To assess the effects of earlier vs. later re-initiation of enteral feeds after necrotizing enterocolitis (NEC). Study design We reviewed the literature to assess timing of enteral feeding after NEC using fixed effects models. Results Three studies met inclusion criteria; no randomized trials. After removal of Bell's Stage I infants, the earlier refeeding group ( $<5-7$ or median 4 days) included 79 infants and later refeeding group ( $\geq 5-7$ or median 10 days) included 119 infants. Pooled analysis revealed earlier re-initiation reduced the incidence in the composite outcome of recurrent NEC and/or post-NEC stricture $(\mathrm{OR}=0.27 ; 95 \% \mathrm{Cl}=0.10-0.75 ; p=0.012)$. Individually, $\mathrm{NEC}$ recurrence (pooled $\mathrm{OR}=0.34$; $95 \% \mathrm{Cl}=0.09-1.29 ; p=0.112)$ or stricture $(\mathrm{OR}=0.34 ; 95 \% \mathrm{Cl}=0.09-1.26 ; p=1.06)$ did not differ between groups.

Conclusions There was no increase in negative outcomes with earlier refeeding after NEC. Earlier initiation of enteral feeds resulted in a significantly lower risk for the combined outcome of recurrent NEC and/or post-NEC stricture.
\end{abstract}

\section{Introduction}

Necrotizing enterocolitis (NEC) is a severe inflammatory disease of the neonatal gut often with devastating consequences. NEC was first described in 1823 in Paris [1] yet still remains one of the most significant causes of morbidity and mortality among extremely premature infants today.

Supplementary information The online version of this article (https:// doi.org/10.1038/s41372-020-0722-1) contains supplementary material, which is available to authorized users.

$\triangle$ Aaron P. Lesher

leshera@musc.edu

1 Department of Pediatrics (Neonatology), Shawn Jenkins Children's Hospital, Charleston, SC, USA

2 Department of Public Health Sciences, Medical University of South Carolina, Charleston, SC, USA

3 Medical University of South Carolina Libraries, Charleston, SC, USA

4 Department of Surgery (Pediatric Surgery), Shawn Jenkins Children's Hospital, Charleston, SC, USA

5 Department of Pediatrics (Neonatology), Rainbow Babies and Children's Hospital, Case Western Reserve University, Cleveland, $\mathrm{OH}$, USA
Advances in neonatal care have decreased many prematurity-related complications; however, the mortality and morbidity of NEC remains largely unchanged. NEC still occurs in $5-22 \%$ of all infants weighing $<1000 \mathrm{~g}$ [2] and the mortality rates range from 35 to $50 \%$ [3]. Despite years of basic science and clinical research, the pathophysiology remains unclear. Experts have concluded that NEC is a complex, multifactorial process likely related to immaturity, inflammation, and feeding typically presenting with abdominal distension, feeding intolerance, bloody stools, and signs of infection. This has historically made providers relatively cautious when re-initiating enteral feeds. Experts and several guidelines have suggested that enteral feedings should be held for 7-14 days [4, 5] to allow for gastrointestinal rest in medically-treated NEC due to concerns for NEC recurrence, post-NEC stricture, or death. However, these guidelines have no scientific evidence behind the length of withholding feeds post-NEC, and the practice of delaying enteral feeds could be harmful. The importance of enteral feeding on intestinal growth has been well established. Blood flow to the gut is higher during enteral feeding allowing for the prevention of atrophy and stimulation of intestinal motility and growth $[6,7]$. Prolonged withholding of enteral feedings carries the risk of extended need for parenteral nutrition, which is associated with infectious and metabolic risks [8]. 
Therefore, it is important to start enteral feeding as soon as safely possible.

Currently, there are no prospective studies supporting the timing of re-initiation of enteral feeds after a diagnosis of medical NEC. Given this lack of conclusive evidence for when to initiate feeds after a diagnosis of NEC, we performed a systematic review and meta-analysis that compares earlier vs. later re-initiation of enteral feedings and the effect on recurrent NEC, post-NEC stricture or both, as a composite outcome. We focused specifically on adverse outcomes because they are typically the barrier considered by the clinical team for deciding when to re-initiate enteral feeds. We hypothesized that a meta-analysis of the combined data from all available studies would demonstrate a lower risk of recurrent NEC and/or post-NEC stricture when enteral feeds are initiated earlier after a diagnosis of NEC.

\section{Methods}

The study protocol for this systematic review and meta-analysis was registered with PROSPERO, \#CRD42019127721. The meta-analysis is reported according to the Preferred Reporting Items for Systematic Reviews and Meta-Analyses (PRISMA) statement [9] (Fig. 1).

\section{Data sources}

To identify studies for inclusion in this review, a Research Informationist with expertize in conducting systematic reviews developed the detailed search strategies in the following databases:

(1) PubMed (U.S. National Library of Medicine, National Institutes of Health)

(2) Scopus (Elsevier)

(3) Cumulative Index to Nursing and Allied Health Literature (CINAHL) Complete (EBSCOhost)

(4) Cochrane Library: Cochrane Database of Systematic Reviews (Wiley)

The search strategies used a combination of subject headings (e.g., MeSH in Pubmed) and keywords for the following three concepts: NEC, enteral nutrition, and timing. The PubMed search strategy was modified for the other three databases, replacing $\mathrm{MeSH}$ terms with appropriate subject headings, when available, and maintaining similar keywords. The search strategies for each database are detailed in Supplementary Information Table 1. The databases were searched from inception through January 16, 2019. No publication dates or language restrictions were applied. To identify additional articles, authors scanned
Fig. 1 PRISMA flow diagram. Flowchart illustrating the search method used to identify studies to be included. Three studies met the inclusion criteria and were included in this metaanalysis.

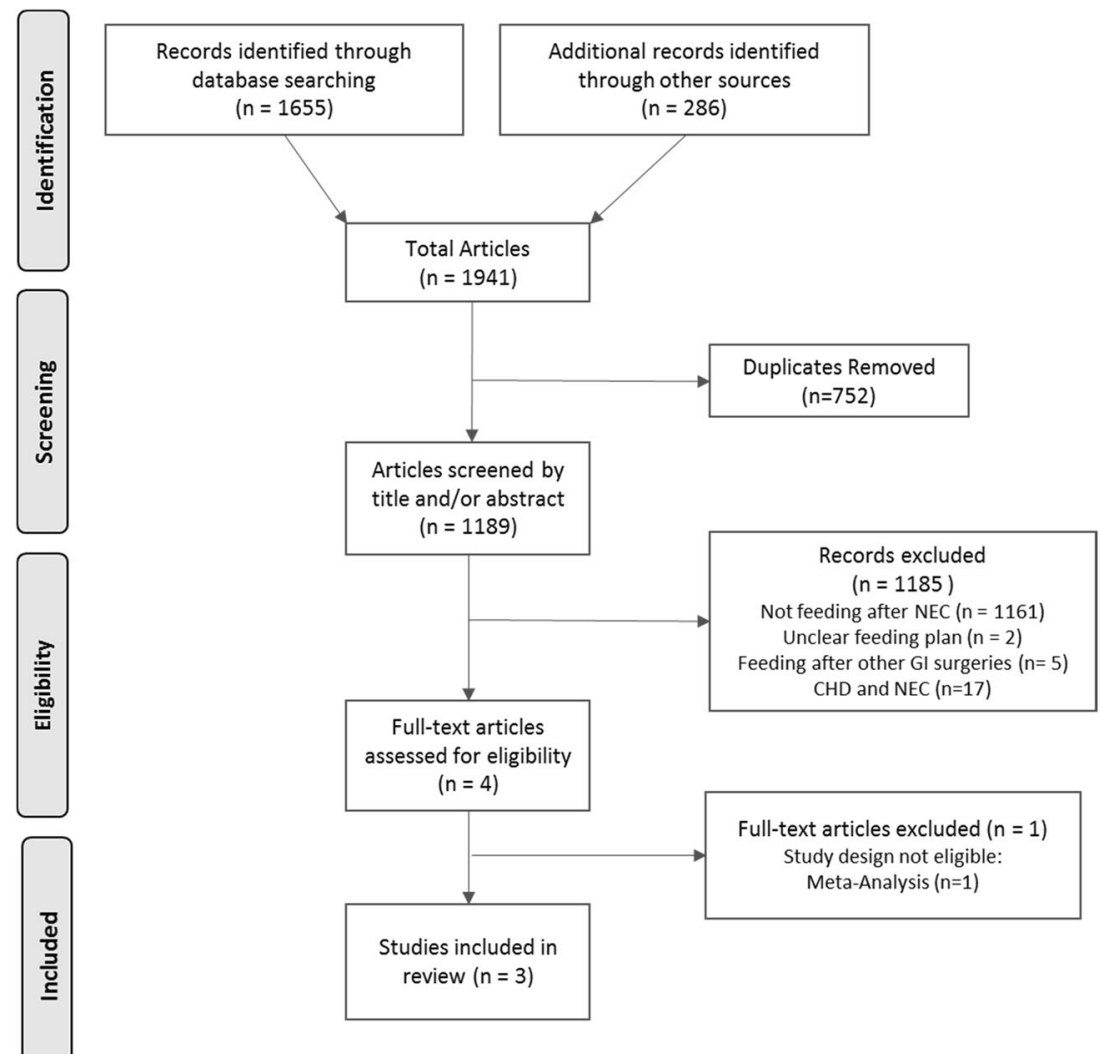


Cochrane Central Register of Controlled Trials, ClinicalTria 1s.gov, Google Scholar, and national conference abstracts. The reference lists of relevant and cited articles were hand searched. References were uploaded to EndNote and screened for relevance.

\section{Study selection}

Two authors (EUP and APL) independently screened the titles and abstracts of all identified studies by using the selection criteria. Inclusion criteria were met if studies reported enteral feeding after NEC diagnosis, a comparison of earlier vs. later enteral feeding, and an evaluation of clinical outcomes. Exclusion criteria were unclear refeeding timing and inclusion of infants with congenital heart disease only. The authors felt that NEC diagnosed with a concomitant congenital heart disease represented a distinct entity from NEC-associated with prematurity. Subsequently, reviewers independently assessed eligibility of the full texts of publications for potentially eligible studies. Records were screened based on title and abstract prior to the full-text articles being evaluated. Disagreements were resolved with discussion.

Our outcomes of interest were:

(1) NEC Stage based on modified Bell's staging criteria $[10,11]$,

(2) Timing of enteral feed initiation after diagnosis of NEC,

(3) Recurrent NEC,

(4) Post-NEC stricture,

(5) Central line-associated bloodstream infection (CLABSI), and

(6) Time to full enteral feeds

The primary outcome in this systematic review was the composite outcome of recurrent NEC and/or post-NEC stricture. Secondary outcomes included CLABSI and time to full enteral feeds. Recurrent NEC, post-NEC stricture, and CLABSI as defined within each study were incorporated within the meta-analysis. If information was incomplete, authors attempted to contact original study's authors. We initially analyzed data as reported in the original articles. However, because Bell's Stage I NEC infants can confound study results, we repeated the metaanalysis with the elimination of these infants from all analyses. Since the only study with Stage I infants was done by author's institution [12], we were able to remove these infants from the original data and present the analysis without Bell's Stage I NEC infants. Analysis using the original data including Stage I infants is presented in Supplementary Information Fig. 1 but did not change the results.

\section{Data extraction and risk of bias assessment}

We extracted the following data:

(1) General study information (author's names, publication year, and study design),

(2) Population-related information (NEC Stage, gestational age),

(3) Feeding details (time of initiation, feeding protocol, and type of milk feed), and

(4) Outcomes of interest as listed above

We assessed the risk of bias using the Newcastle-Ottawa Scale [13] for these observational clinical studies.

\section{Data synthesis and statistical methods}

We performed the systematic review according to the PRISMA statement. Aggregate count data were analyzed using Stata software version 13 (StataCorp, College Station, TX) to obtain a summary estimate for the pooled data. Statistical heterogeneity was determined using the Cochran's Q statistic and I [2]. We used a fixed effects model for the meta-analysis for all outcomes since we found no evidence for heterogeneity. Both effect models gave very similar odds ratios, but the confidence intervals were slightly broader with the random effects model. Given the similarity between the results and since the studies share common interventions and outcomes, we used a fixed effects model. Sample size and aggregate level data did not allow us to assess assumptions of normality or variance. $P$ values of $\leq 0.05$ were considered statistically significant. Secondary outcomes were described, and student's $t$ tests were used to evaluate statistically significant differences between earlier and later feeding.

\section{Results}

\section{Study characteristics}

We identified 1941 titles and abstracts through our literature search with 1189 unique records after duplicates were removed (Fig. 1). Those records were reviewed, based on title and abstract, and only four proved to be potentially eligible for full-text evaluations. No randomized controlled trials were identified. Three retrospective or change in practice studies $[12,14,15]$ and one meta-analysis [16] met our inclusion criteria. A previous meta-analysis by Hock et al. [16] included only two of the three studies we identified as eligible for this current meta-analysis. The third singlecenter study [12] was published after the Hock et al. [16] meta-analysis. The Hock et al. meta-analysis included a total 
of 91 patients with 56 infants in the earlier enteral feeding group after NEC diagnosis and 35 infants in the later feeding group. The addition of the third study more than doubled the total cohort and resulted in 229 subjects in a combined analysis (Table 1). A description of our quality assessment is presented in Supplementary Information Table 2.

Bohnhorst et al. [14] studied all infants <36 weeks' gestational age diagnosed with Bell's Stage II or greater (both medically and surgically treated) in a neonatal intensive care unit (NICU) in Germany. This study involved a practice change in which providers re-initiated feeds after there was no evidence of portal vein gas on abdominal ultrasound for 3 consecutive days. The earlier feeding group started refeeding at a median of 4 days $(n=26)$ after NEC diagnosis. The later feeding group, prior to the practice change, had feeds re-initiated at a median of 10 days which was based on clinician discretion $(n=18)$. Brotschi et al. [15] performed a multicentered, retrospective cohort study in Switzerland that included NEC Bell's Stage II only (no surgical cases) with a cut-off of 5 days, traditionally half the longest standard regimen of 10 days among these institutions. The earlier group included 30 infants with feeds reinitiated $<5$ days after NEC diagnosis, and the later group included 17 infants for whom re-initiation of enteral feeds occurred $>5$ days after diagnosis, again at clinician discretion. Arbra et al. [12] from South Carolina published a retrospective study including all infants with NEC Bell's Stage IIIA or less (no surgical cases but included infants with Stage I). In their original analysis, they included 138 infants, however 31 of these infants were diagnosed with Bell's Stage I NEC and were removed for subsequent analysis at the request of the reviewers. The authors noted a wide practice variation at their institution in which some nonsurgical NEC patients underwent earlier refeeding and others later refeeding. This inconsistency allowed them to choose an arbitrary cut-off of 7 days to differentiate the infants who were fed earlier from those that were fed in the more traditional later fasting period. This study found 40 infants in the earlier refeeding group (re-initiation of feeds $<7$ days after the onset of NEC) and 98 infants in the later refeeding group ( $\geq 7$ days). With the three studies combined, there are a total of 229 infants, 96 infants in the earlier reinitiation of enteral feeding group $(<5-7$ days or median 4 days), and 133 infants in the later feeding group ( $\geq 5-7$ days or median 10 days). All studies evaluated incidences of recurrent NEC and post-NEC stricture (Supplementary Information Table 3). After removal of Stage I infants, the earlier enteral feeding group from the Arbra study [12] had 23 infants in the earlier refeeding group and 84 infants in the later refeeding group. Then with the three studies combined, there was a total of 198 infants, 79 infants in the earlier re-initiation of enteral feeding group and 119 infants in the later feeding group (Table 2).
Primary outcomes-recurrent NEC and/or post-NEC stricture

The primary outcome was the incidence of recurrent NEC and/or post-NEC stricture as a composite outcome, given that both outcomes are rare but clinically relevant. There was a significant benefit to earlier re-initiation of enteral feeding after NEC diagnosis (pooled $\mathrm{OR}=0.27 ; 95 \% \mathrm{Cl}=$ $0.10-0.75 ; p=0.012)$ when the composite outcome of recurrent NEC and/or post-NEC stricture was evaluated (Fig. 2a). Recurrent NEC (pooled OR $=0.34 ; 95 \% \mathrm{Cl}=$ 0.09-1.29; $p=0.112$ ) (Fig. 2b) or post-NEC stricture (pooled $\mathrm{OR}=0.34 ; \quad 95 \% \quad \mathrm{Cl}=0.09-1.26 ; \quad p=0.106$ ) (Fig. 2c) did not differ between earlier and later groups as individual outcomes in the composite analysis. Results were very similar when all infants (including Bell's Stage I NEC) within the original data were included [Composite outcome (pooled $\mathrm{OR}=0.32 ; 95 \% \quad \mathrm{Cl}=0.13-0.78 ; \quad p=0.013$ ); recurrent $\mathrm{NEC}$ (pooled $\mathrm{OR}=0.46 ; 95 \% \mathrm{Cl}=0.15-1.40$; $p=0.170$ ); post-NEC stricture (pooled OR $=0.28 ; 95 \%$ $\mathrm{Cl}=0.07-1.02 ; \quad p=0.053$ ) (Supplementary Information Fig. 1).

\section{Secondary outcomes}

All three studies evaluated time to reach full enteral feeds following re-initiation of feeding after a NEC diagnosis. Each study had a slightly different method of advancing enteral feeds. However, when pooled, the mean time to full feeds after NEC diagnosis was significantly lower in the earlier group compared with the later group (11.6 \pm 2.9 days vs. $16.2 \pm 4.5$ days, $p<0.001, t$-test) (Table 2) with similar results when Stage I infants were included (Supplementary Information Table 3). This suggested that an earlier start to refeeding after NEC did not hamper the rate of increase in enteral feeding. The incidence of CLABSI was $7.6 \%$ in the earlier group and $16 \%$ in the later group (similar when Stage I infants were still included) with a pooled $\mathrm{OR}=0.31$ (95\% $\mathrm{Cl}=0.11-0.86 ; p=0.024)$ (Fig. 2d) showing a significant benefit to earlier re-initiation of enteral feeds after NEC diagnosis.

\section{Discussion}

The relationship between enteral feeding and the development of NEC was discovered in the 1970s, resulting in delayed feeding as standard therapy after NEC in many NICUs across the world. However, this practice is not evidence based and may be harmful. This meta-analysis demonstrated a significant benefit to earlier initiation of enteral feeding when evaluating for the composite outcome of recurrent NEC and/or post-NEC stricture, as well as a 


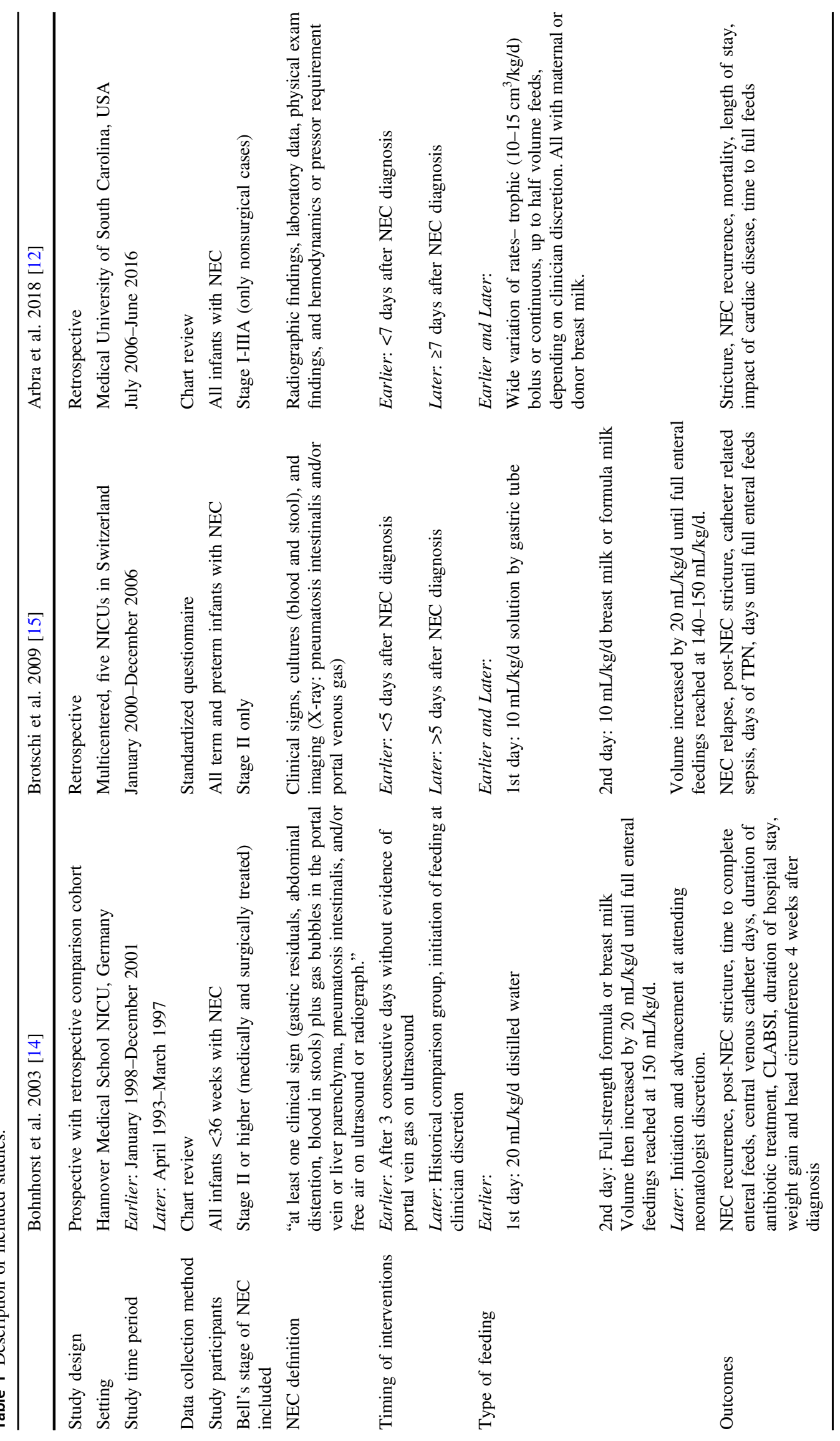




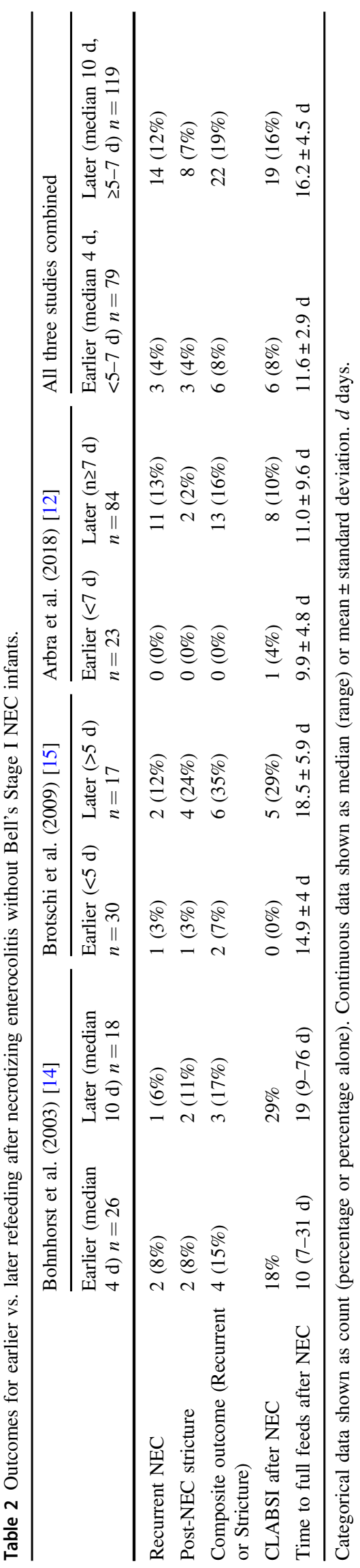

decreased time interval to full feeds and incidence of CLABSI.

When Frantz et al. [17] reported that reestablishing feeds $<10$ days after NEC was associated with disease recurrence in "several patients" in 1975, it is likely that this spurred the standard practice that all enteral feedings should be held for 7-14 days in medically-treated NEC. However, this study had no quantitative information for this recurrence risk. Later in 1993, Stringer et al. [18] described a cohort of 12 infants with recurrent NEC and found no consistent association with type or timing of enteral feeds. Recent research now supports the practice that early initiation of enteral feeds may be safe and also reduce other co-morbidities associated with prematurity. A recent Cochrane systematic review observed that delayed introduction of enteral feeds in routine general care of the very preterm or low birth weight infant did not reduce the risk of NEC or all-cause mortality [19], nor did it improve feeding intolerance or growth rates [20]. Therefore, with the evidence supporting earlier introduction of enteral feedings generally, we hypothesized that re-initiating enteral feeding after diagnosis of NEC earlier could be beneficial as well.

During the initial management of NEC, there is no debate that enteral feeding should be held for a period of time. However, given the intrinsic benefits of trophic enteral nutrition [6, 21], feeds should be re-initiated as soon as safely possible. Currently, the literature lacks compelling evidence for when the re-initiation of enteral feeds should occur. The initial meta-analysis done by Hock et al. [16] included a total of 91 infants from two non-randomized studies and suggested some trends toward a benefit to earlier refeeding. However, there was not a sufficient number of subjects to reach statistical significance. With the inclusion of the third, and larger, retrospective study, there is a more robust population of 229 infants (198 without Stage I infants) to analyze the effects of clinician-determined earlier vs. later re-initiation of enteral feeds. Our meta-analysis, using the data as reported with and without including Bell's Stage I infants confound the results, demonstrated a significant benefit to earlier initiation of enteral feeds with respect to the composite outcome of recurrent NEC and/or post-NEC stricture. There was not a statistically significant benefit demonstrated for these individual outcomes, although both outcomes favored earlier refeeding. The lack of significance may be due to the small subject numbers.

The incidence of recurrent NEC and of post-NEC strictures are serious complications and likely influence clinicians to withhold re-initiation of feeding. The recurrence rates among the combined studies (including Stage I infants) was $5.2 \%$ in the earlier group and $11.3 \%$ in the later group, which is similar to the overall recurrence rates of $6-10 \%[18,22]$ published by other investigators. Only the study by Brotschi et al. [15] mentioned the timing of 


\section{A. Composite Outcome of Recurrent NEC and/or Post-NEC Stricture}

\begin{tabular}{|c|c|c|c|c|c|}
\hline Study (Author Year) & $\begin{array}{l}\text { Earlier } \\
\mathrm{n} / \mathrm{N}\end{array}$ & $\begin{array}{c}\text { Later } \\
\mathrm{n} / \mathrm{N}\end{array}$ & Odds of Recurrence and/or Stricture & Odds Ratio $(95 \% \mathrm{Cl})$ & $\%$ Weight \\
\hline Bohnhorst 2003 & $4 / 26$ & $3 / 18$ & & $0.91(0.18,4.66)$ & 18.79 \\
\hline Brotschi 2009 & $2 / 30$ & $6 / 17$ & & $0.13(0.02,0.75)$ & 44.76 \\
\hline Arbra 2018 & $0 / 23$ & $13 / 84$ & & $0.11(0.01,1.97)$ & 36.45 \\
\hline Overall $(p=0.012)$ & $6 / 79$ & $22 / 119$ & & $0.27(0.10,0.75)$ & 100.00 \\
\hline$(\mathrm{Q}=3.14$ on $2 \mathrm{df}, \mathrm{p}=0.208)$ & & & & & \\
\hline
\end{tabular}

\section{B. Recurrent NEC}

\begin{tabular}{|c|c|c|c|c|c|}
\hline Study (Author Year) & $\begin{array}{c}\text { Earlier } \\
\mathrm{n} / \mathrm{N}\end{array}$ & $\begin{array}{l}\text { Later } \\
\mathrm{n} / \mathrm{N}\end{array}$ & Odds of Recurrence & Odds Ratio (95\% Cl) & $\%$ Weight \\
\hline Bohnhorst 2003 & $2 / 26$ & $1 / 18$ & + & $1.42(0.12,16.91)$ & 12.81 \\
\hline Brotschi 2009 & $1 / 30$ & $2 / 17$ & & $0.26(0.02 .3 .09)$ & 28.98 \\
\hline Arbra 2018 & $0 / 23$ & $11 / 84$ & & $0.14(0.01,2.40)$ & 58.22 \\
\hline Overall $(p=0.112)$ & $3 / 79$ & $14 / 119$ & 一 & $0.34(0.09,1.29)$ & 100.00 \\
\hline$(Q=1.72$ on $2 d f ; p=0.423)$ & & & & & \\
\hline
\end{tabular}

\section{Post-NEC Stricture}

\begin{tabular}{|c|c|c|c|c|c|}
\hline Study (Author Year) & $\begin{array}{c}\text { Earlier } \\
\mathrm{n} / \mathrm{N}\end{array}$ & $\begin{array}{c}\text { Later } \\
\mathrm{n} / \mathrm{N}\end{array}$ & Odds of Stricture & Odds Ratio $(95 \% \mathrm{Cl})$ & $\%$ Weight \\
\hline Bohnhorst 2003 & $2 / 26$ & $2 / 18$ & $\frac{1}{1}$ & $0.67(0.09,5.23)$ & 26.62 \\
\hline Brotschi 2009 & $1 / 30$ & $4 / 17$ & $\frac{1}{1}$ & $0.11(0.01 .1 .10)$ & 60.23 \\
\hline Arbra 2018 & $0 / 23$ & $2 / 84$ & $\frac{1}{1}$ & $0.70(0.03,15.14)$ & 13.15 \\
\hline Overall $(p=0.106)$ & $3 / 79$ & $8 / 119$ & & $0.34(0.09,1.26)$ & 100.00 \\
\hline \multicolumn{6}{|l|}{$(Q=1.53$ on $2 \mathrm{df} ; \mathrm{p}=0.465)$} \\
\hline
\end{tabular}

\section{CLABSI}

\begin{tabular}{|c|c|c|c|c|c|}
\hline Study (Author Year) & $\begin{array}{c}\text { Earlier } \\
\mathrm{n} / \mathrm{N}\end{array}$ & $\begin{array}{l}\text { Later } \\
\mathrm{n} / \mathrm{N}\end{array}$ & Odds of CLABSI & Odds Ratio $(95 \% \mathrm{Cl})$ & $\%$ Weight \\
\hline Bohnhorst 2003 & $5 / 26$ & $5 / 18$ & & $0.62(0.15,2.56)$ & 32.01 \\
\hline Brotschi 2009 & $0 / 30$ & $5 / 17$ & & $0.04(0.00 .0 .73)$ & 45.92 \\
\hline Arbra 2018 & $1 / 23$ & $8 / 84$ & & $0.43(0.05,3.64)$ & 22.06 \\
\hline Overall $(p=0.024)$ & $6 / 79$ & $18 / 119$ & & $0.31(0.11,0.86)$ & 100.00 \\
\hline$(\mathrm{Q}=2.96$ on $2 \mathrm{df} ; \mathrm{p}=0.228)$ & & & & & \\
\hline
\end{tabular}

Fig. 2 Forest Plots of 3 studies. Earlier vs. later re-initiation of enteral feeding without Bell's Stage I NEC infants on composite outcome of recurrent NEC and/or post-NEC stricture (a), recurrent NEC (b), post-NEC stricture (c), and CLABSI (d). Cl, confidence interval.

recurrence from initial NEC episode, which was after full enteral feedings were reestablished, therefore it is unclear if recurrence was related to when enteral feeds were re- intiated. In all three studies [12, 14, 15] included in the meta-analysis, the odds of a CLABSI were significantly lower and full feedings were achieved significantly earlier 
in the earlier refeeding group once feeds were restarted. The earlier refeeding group overall had a lower incidence of recurrence, although not statistically significant compared with the later refeeding group. All of the studies included used a standard feeding protocol involving a slow advancement in feeds. Given that standardized feeding protocols have been shown to reduce the incidence of NEC [23], this may have contributed to the low adverse outcome rates despite the earlier and later re-initiation of feeding.

Post-NEC intestinal strictures are a well-known complication affecting up to $25 \%$ of infants with proven NEC $[24,25]$. A prolonged fasting period presumably prevents the ischemic-reperfusion injury thought to cause intestinal strictures [26]; however, infants who were refed earlier in our cohort did not have higher rates of post-NEC stricture. This trend was true in all three studies included in the metaanalysis. The total incidence of stricture was $5.7 \%$, although one study [14] did include surgical infants, which could possibly be underrepresenting the general population, but the incidence of stricture was similar amongst the three studies.

The primary strength of this meta-analysis is that the number of infants included in the study doubled compared with the prior meta-analysis. The meta-analysis addresses a critical problem in clinical care of the premature infant with NEC. With the increase in sample size, we were able to demonstrate a significant difference for important adverse outcomes. The limitations to this meta-analysis are primarily related directly to the inherent limitations of the three included studies. These limitations include the lack of randomized treatment group assignments, which increases the possible bias of less sick infants starting enteral feeds earlier after NEC diagnosis. Two of the studies [12, 15] used clinician discretion as to the timing of restarting enteral feeds, which likely placed infants who were less sick in the earlier feeding group possibly contributing to less adverse outcomes in that group. However, the Bohnhorst et al. study [14] used lack of portal vein gas on ultrasound for 3 days to prospectively determine when feeding should be started. There are several confounders that not all of the studies addressed. These included, but not limited to, the degree of ventilator support, hemodynamic stability, and length of antibiotics which could have additionally. contributed to the clinical decision of earlier vs. later refeeding groups. Populations also varied among these studies. Only one study included surgical cases and only one study included Bell's Stage I infants. While surgical cases can skew how long enteral feeds are held, we were not able to obtain original data from those authors to be able to remove surgically-treated infants from the analysis. However, we were able to remove Stage I infants, which did not significantly alter results which is likely why we found low heterogeneity in the meta-analysis model.
Our purpose in performing this study was not necessarily to show that earlier feeding was "better" but to challenge the dogma that earlier re-initiation of enteral feedings after NEC was harmful. Several institutions (including ours) have already implemented earlier refeeding protocols, and hopefully we can gain more insight as these project results are reported.

\section{Conclusion}

In this meta-analysis of three previously published studies of almost 200 premature infants, there was no increase in negative outcomes with earlier enteral refeeding after NEC. Earlier refeeding resulted in a significantly lower risk for the combined outcome of recurrent NEC and/or post-NEC stricture suggesting that it is safe and may be preferable to start enteral feeds $<7$ days after a NEC diagnosis. Although a data-driven consensus on the exact timing remains elusive, the incidence of CLABSI was significantly lower and time to full enteral feedings was achieved significantly sooner in the earlier refeeding group. There was no statistically significant increase in rates of recurrent NEC or postNEC stricture with earlier refeeding. However, these findings are only based on observational retrospective studies; a multicentered randomized control trial would be necessary to minimize potential treatment biases. Currently, this metaanalysis presents the largest cohort available to help address the ideal timing of enteral feeding after NEC.

\section{Compliance with ethical standards}

Conflict of interest The authors declare that they have no conflict of interest.

Publisher's note Springer Nature remains neutral with regard to jurisdictional claims in published maps and institutional affiliations.

\section{References}

1. Beckwith JB. Charles-Michel Billard (1800-1832): pioneer of infant pathology. Pediatric and developmental pathology: the official journal of the Society for Pediatric Pathology and the Paediatric Pathology. Society. 2002;5:248-56.

2. Battersby C, Santhalingam T, Costeloe K, Modi N. Incidence of neonatal necrotising enterocolitis in high-income countries: a systematic review. Arch Dis Child Fetal neonatal Ed. 2018;103: F182-f9.

3. Dimmit RA, Moss RL. Clinical management of necrotizing enterocolitis. NeoReviews. 2001;2:110-7.

4. King C. Enteral feeding. In: Jones EaK, Caroline, editors. Feeding and nutrition in the preterm infant. Philadelphia, PA: Elsevier; 2005. p. 104.

5. Neu J, Walker WA. Necrotizing enterocolitis. N Engl J Med. 2011;364:255-64.

6. Niinikoski H, Stoll B, Guan X, Kansagra K, Lambert BD, Stephens $\mathrm{J}$, et al. Onset of small intestinal atrophy is associated with 
reduced intestinal blood flow in TPN-fed neonatal piglets. J Nutr. 2004;134:1467-74.

7. Embleton ND, Zalewski SP. How to feed a baby recovering from necrotising enterocolitis when maternal milk is not available. Arch Dis Child Fetal neonatal Ed. 2017;102:F543-f6.

8. Moss RL, Kalish LA, Duggan C, Johnston P, Brandt ML, Dunn JC, et al. Clinical parameters do not adequately predict outcome in necrotizing enterocolitis: a multi-institutional study. J Perinatol. 2008;28:665-74.

9. Moher D, Liberati A, Tetzlaff J, Altman DG. Preferred reporting items for systematic reviews and meta-analyses: the PRISMA statement. PLoS Med. 2009;6:e1000097.

10. Bell MJ, Ternberg JL, Feigin RD, Keating JP, Marshall R, Barton L, et al. Neonatal necrotizing enterocolitis. Therapeutic decisions based upon clinical staging. Ann Surg. 1978;187:1-7.

11. Walsh MC, Kliegman RM. Necrotizing enterocolitis: treatment based on staging criteria. Pediatr Clin North Am. 1986;33:179-201.

12. Arbra CA, Oprisan A, Wilson DA, Ryan RM, Lesher AP. Time to reintroduction of feeding in infants with nonsurgical necrotizing enterocolitis. J Pediatr Surg. 2018;53:1187-91.

13. Wells G, Shea B, O'Connell D, Peterson J, Welch V, Losos M, et al. The Newcastle-Ottawa Scale (NOS) for assessing the quality of nonrandomised studies in meta-analysis 2011. http://www.ohri. ca/programs/clinical_epidemiology/oxford.asp.

14. Bohnhorst B, Muller S, Dordelmann M, Peter CS, Petersen C, Poets CF. Early feeding after necrotizing enterocolitis in preterm infants. J Pediatrics. 2003;143:484-7.

15. Brotschi B, Baenziger O, Frey B, Bucher HU, Ersch J. Early enteral feeding in conservatively managed stage II necrotizing enterocolitis is associated with a reduced risk of catheter-related sepsis. J Perinat Med. 2009;37:701-5.
16. Hock AM, Chen Y, Miyake H, Koike Y, Seo S, Pierro A. Initiation of enteral feeding after necrotizing enterocolitis. Eur $\mathrm{J}$ Pediatr Surg. 2018;28:44-50.

17. Frantz ID III, L'Heureux P, Engel RR, Hunt CE. Necrotizing enterocolitis. J Pediatrics. 1975;86:259-63.

18. Stringer MD, Brereton RJ, Drake DP, Kiely EM, Capps SN, Spitz L. Recurrent necrotizing enterocolitis. J Pediatr Surg. 1993;28:979-81.

19. Morgan J, Young L, McGuire W. Delayed introduction of progressive enteral feeds to prevent necrotising enterocolitis in very low birth weight infants. Cochrane Database Syst Rev. 2014;12:Cd001970.

20. Morgan J, Bombell S, McGuire W. Early trophic feeding versus enteral fasting for very preterm or very low birth weight infants. Cochrane Database Syst Rev. 2013;3:Cd000504.

21. Lucas A, Bloom SR, Aynsley-Green A. Gut hormones and 'minimal enteral feeding'. Acta Paediatrica Scandinavica. 1986;75:719-23.

22. Thyoka M, Eaton S, Hall NJ, Drake D, Kiely E, Curry J, et al. Advanced necrotizing enterocolitis part 2: recurrence of necrotizing enterocolitis. Eur J Pediatr Surg. 2012;22:13-6.

23. Jasani B, Patole S. Standardized feeding regimen for reducing necrotizing enterocolitis in preterm infants: an updated systematic review. J Perinatol. 2017;37:827-33.

24. Schwartz MZ, Hayden CK, Richardson CJ, Tyson KR, Lobe TE. A prospective evaluation of intestinal stenosis following necrotizing enterocolitis. J Pediatr Surg. 1982;17:764-70.

25. Thompson AM, Bizzarro MJ. Necrotizing enterocolitis in newborns: pathogenesis, prevention and management. Drugs 2008;68:1227-38.

26. Crafts TD, Hunsberger EB, Jensen AR, Rescorla FJ, Yoder MC, Markel TA. Direct peritoneal resuscitation improves survival and decreases inflammation after intestinal ischemia and reperfusion injury. J Surgical Res. 2015;199:428-34. 